\title{
Honeysuckle-encoded atypical microRNA2911 directly targets influenza A viruses
}

Zhen Zhou ${ }^{1, *}$, Xihan $\mathrm{Li}^{1, *}$, Jinxiong Liu ${ }^{2, *}$, Lei Dong ${ }^{1,}{ }^{*}$, Qun Chen ${ }^{1}$, Jialing Liu ${ }^{1}$, Huihui Kong ${ }^{2}$, Qianyi Zhang ${ }^{2}$, Xian $\mathrm{Qi}^{3}$, Dongxia Hou ${ }^{1}$, Lin Zhang ${ }^{1}$, Guoquan Zhang ${ }^{2}$, Yuchen Liu ${ }^{1}$, Yujing Zhang ${ }^{1}$, Jing Li $^{1}$, Jin Wang ${ }^{1}$, Xi Chen ${ }^{1}$, Hua Wang ${ }^{3}$, Junfeng Zhang ${ }^{1}$, Hualan Chen ${ }^{2}$, Ke Zen ${ }^{1}$, Chen-Yu Zhang ${ }^{1}$

${ }^{1}$ Jiangsu Engineering Research Center for MicroRNA Biology and Biotechnology, State Key Laboratory of Pharmaceutical Biotechnology, School of Life Sciences, Nanjing University, Nanjing, Jiangsu 210093, China; ${ }^{2}$ State Key Laboratory of Veterinary Biotechnology, Harbin Veterinary Research Institute, Chinese Academy of Agricultural Sciences, Harbin, Heilongjiang 150001, China $;{ }^{3}$ Jiangsu Provincial Center for Disease Control and Prevention, Nanjing, Jiangsu 210090, China

Influenza A viruses (IAVs), particularly H1N1, H5N1 and H7N9, pose a substantial threat to public health worldwide. Here, we report that MIR2911, a honeysuckle (HS)-encoded atypical microRNA, directly targets IAVs with a broad spectrum. MIR2911 is highly stable in HS decoction, and continuous drinking or gavage feeding of HS decoction leads to a significant elevation of the MIR2911 level in mouse peripheral blood and lung. Bioinformatics prediction and a luciferase reporter assay showed that MIR2911 could target various IAVs, including H1N1, H5N1 and H7N9. Synthetic MIR2911 significantly inhibited H1N1-encoded PB2 and NS1 protein expression, but did not affect mutants in which the MIR2911-binding nucleotide sequences were altered. Synthetic MIR2911, extracted RNA from HS decoction and HS decoction all significantly inhibited H1N1 viral replication and rescued viral infection-induced mouse weight loss, but did not affect infection with a mutant virus in which the MIR2911-binding nucleotide sequences of $P B 2$ and NS1 were altered. Importantly, the inhibitory effect of HS decoction on viral replication was abolished by an anti-MIR2911 antagomir, indicating that the physiological concentration of MIR2911 in HS decoction could directly and sufficiently suppress H1N1 viral replication. MIR2911 also inhibited H5N1 and H7N9 viral replication in vitro and in vivo. Strikingly, administration of MIR2911 or HS decoction dramatically reduced mouse mortality caused by H5N1 infection. Our results demonstrate that MIR2911 is the first active component identified in Traditional Chinese Medicine to directly target various IAVs and may represent a novel type of natural product that effectively suppresses viral infection.

Keywords: microRNA; influenza A virus; H1N1; H5N1; H7N9; honeysuckle; MIR2911

Cell Research (2015) 25:39-49. doi:10.1038/cr.2014.130; published online 7 October 2014

\section{Introduction}

Influenza A viruses (IAVs) pose a substantial threat to public health worldwide. In the last century, there were

*These four authors contributed equally to this work.

Correspondence: Chen-Yu Zhang ${ }^{\mathrm{a}}$, Ke Zen ${ }^{\mathrm{b}}$, Hualan $\mathrm{Chen}^{\mathrm{c}}$,

Junfeng Zhang ${ }^{\mathrm{d}}$, Hua Wang ${ }^{\mathrm{e}}$

${ }^{a}$ E-mail: cyzhang@nju.edu.cn

bE-mail: kzen@nju.edu.cn

${ }^{c}$ E-mail: chenhualan@caas.cn

${ }^{\mathrm{d} E}$ E-mail: jfzhang@nju.edu.cn

${ }^{e}$ E-mail: hua@jscdc.cn

Received 18 June 2014; revised 28 August 2014; accepted 1 September 2014; published online 7 October 2014 three large influenza pandemics that caused high levels of mortality, including the most serious pandemic in recorded history, the 1918 Spanish flu, which was responsible for more than 50 million human deaths $[1,2]$. The highly pathogenic $\mathrm{H} 5 \mathrm{~N} 1$ subtype and the recently reported H7N9 subtype have shown potential to cause new human influenza pandemics [3-5]. The high mutability of pathogenic IAVs enables them to become resistant to various preventive treatments, including vaccines and antibodies [6]. Honeysuckle (HS, Lonicera japonica), a well-known Chinese herb, has been used to effectively treat influenza infection for thousands of years. Several reports have shown that HS decoction can suppress the replication of influenza virus [7, 8]. However, the active compounds 
in HS decoction and the mechanism by which they block viral replication have remained unclear.

On the other hand, our previous study has shown that MIR168a derived from food plants can pass through the gastrointestinal (GI) tract and enter into the circulation and various organs of mice. In mouse liver, plant MIR168a targets low-density lipoprotein (LDL) receptor adapter protein 1 (LDLRAP1), which results in elevation of LDL-cholesterol level in the plasma [9]. This study opened an avenue for exploring the physiological function of exogenous plant microRNAs (miRNAs) in human and animals. However, the implications of exogenous plant miRNA-mediated cross-kingdom regulation and the mechanisms responsible for plant miRNA absorption and transfer remain largely unknown, highlighting the need for further investigations. Given that the patients with influenza infection were traditionally administered with HS decoction, we hypothesize that plant miRNA in HS decoction is capable of entering the human body through GI tract to exert a therapeutic effect. In the present study, we showed that a plant miRNA enriched in HS decoction, named MIR2911, directly targets various influenza A subtypes both in vitro and in vivo.

\section{Results}

Plant MIR2911 is absorbed and delivered into the lungs in mice after administration of HS decoction or synthetic MIR2911

All the short RNA fragments $(<30 \mathrm{nt})$ extracted from HS were analyzed using Illumina deep-sequencing technology. The results showed that HS contains a panel of unique miRNA fragments (GEO accession number: GSE55268). In total, 562230 reads of 148 miRNAs were obtained from 9676848 total reads; 26 miRNAs had more than 100 reads (Figure 1A and Supplementary information, Table S1). Among these plant miRNAs, MIR166g had the highest copy number (222 051). An HS decoction was prepared by boiling HS in double-distilled water for $30 \mathrm{~min}$ (final concentration: $\sim 0.2 \mathrm{~g} \mathrm{HS} / \mathrm{ml}$ water) and was further analyzed for short RNA fragments. Strikingly, Illumina sequencing analysis revealed that most miRNAs detected in HS except for MIR2911 were degraded during the boiling process (Supplementary information, Figure S1), and MIR2911 represented more than $70 \%$ of the reads of miRNAs in the final HS decoction (Figure 1B). The copy numbers of MIR166g and MIR2914, two miRNAs with high expression in HS, were reduced from 222051 to 315 and 25422 to 706 in the final HS decoction, respectively, while the copy number of MIR2911 remained high (11 322). To verify the Illumina sequencing results, TaqMan probe-based RT-qPCR assays and northern blot analyses were performed. The RT-qPCR results showed that the concentration of MIR2911 in HS was $0.34 \mathrm{pmol} / \mathrm{g}$, while the concentration of MIR2911 in HS decoction $(0.2 \mathrm{~g}$ $\mathrm{HS} / \mathrm{ml}$ ) was $0.06 \mathrm{pmol} / \mathrm{ml}$ (Figure 1C). By referencing the synthetic MIR2911 concentrations (Figure 1D, left panel), the northern blotting results confirmed that the concentrations of MIR2911 in HS and HS decoction (0.2 $\mathrm{g} \mathrm{HS} / \mathrm{ml}$ ) were $\sim 0.40 \mathrm{pmol} / \mathrm{g}$ and $0.08 \mathrm{pmol} / \mathrm{ml}$, respectively (Figure 1D, right panel). Compared with other plant miRNAs or small RNA fragments, MIR2911 may be more resistant to boiling during the preparation of HS decoction. In support of the high stability of MIR2911, MIR2911 was found to be largely intact following RNase treatment (Supplementary information, Figure S2). To test whether the stability of MIR2911 depends on its unique sequence, 5'-GGCCGGGGGACGGACUGGGA-3', two mutants were created by mutating the $5^{\prime}$ GG to $5^{\prime}$-AA or the $3^{\prime}$-GGA to 3'-AAA. The mutants were not resistant to RNase treatments (Supplementary information, Figure S2), suggesting that the stability of MIR2911 is based on its specific sequence and high GC content.

The absorption was assessed in mice administered $500 \mu \mathrm{l}$ of HS decoction (MIR2911 concentration: $\sim 0.12$ $\mathrm{pmol} / \mathrm{ml}$ ) by single gavage feeding. The basal concentration of MIR2911 in mouse plasma was $242.0 \pm 23.8 \mathrm{fM}$; the plasma concentration of MIR2911 increased to 517.1 $\pm 133.6 \mathrm{fM}$ at $3 \mathrm{~h}$, reached a peak of $1189.2 \pm 323.1 \mathrm{fM}$ at $6 \mathrm{~h}$ and decreased to $923.0 \pm 189.3 \mathrm{fM}$ at $12 \mathrm{~h}$ after HS decoction administration (Figure 1E). Figure 1F showed that the level of MIR2911 in mouse lungs continously increased, reached a peak level at $6 \mathrm{~h}$ and decreased to the basal level at $12 \mathrm{~h}$ after administration. Most of the MIR2911 in mouse peripheral blood was detected in the fraction containing cell-derived microvesicles (MVs) (Supplementary information, Figure S3A), implying that the MIR2911 in HS decoction might initially be taken up from the GI tract, repackaged into MVs by intestinal epithelial cells and finally secreted into the circulation. Immunoprecipitation data revealed that the majority of MIR2911 in the MVs derived from mouse peripheral blood was associated with the Argonaute 2 (AGO2) complex (Supplementary information, Figure S3B).

Concentrations of MIR2911 in the plasma and lung were further assessed when mice continually drank HS decoction for 3 days $(0.06 \mathrm{pmol} / \mathrm{ml}, \sim 4 \mathrm{ml} /$ day $)$. Mice that drank sterile water served as controls. The plasma levels of MIR2911 in mice after drinking HS decoction increased by 3 -fold (from $250.5 \pm 38.8 \mathrm{fM}$ to $831.8 \pm$ $47.8 \mathrm{fM}$ ), whereas no change in the MIR2911 level was observed in control mice (from $215.8 \pm 38.2 \mathrm{fM}$ to 253.3 

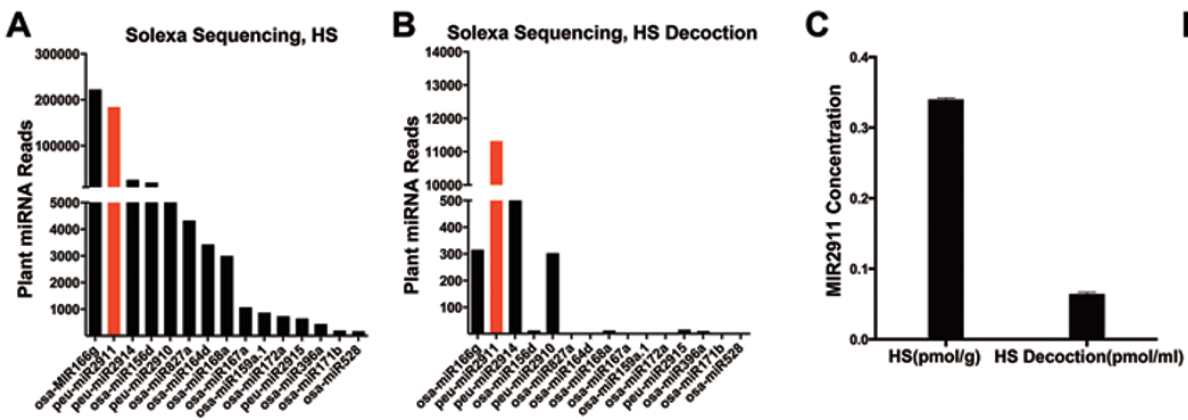

D
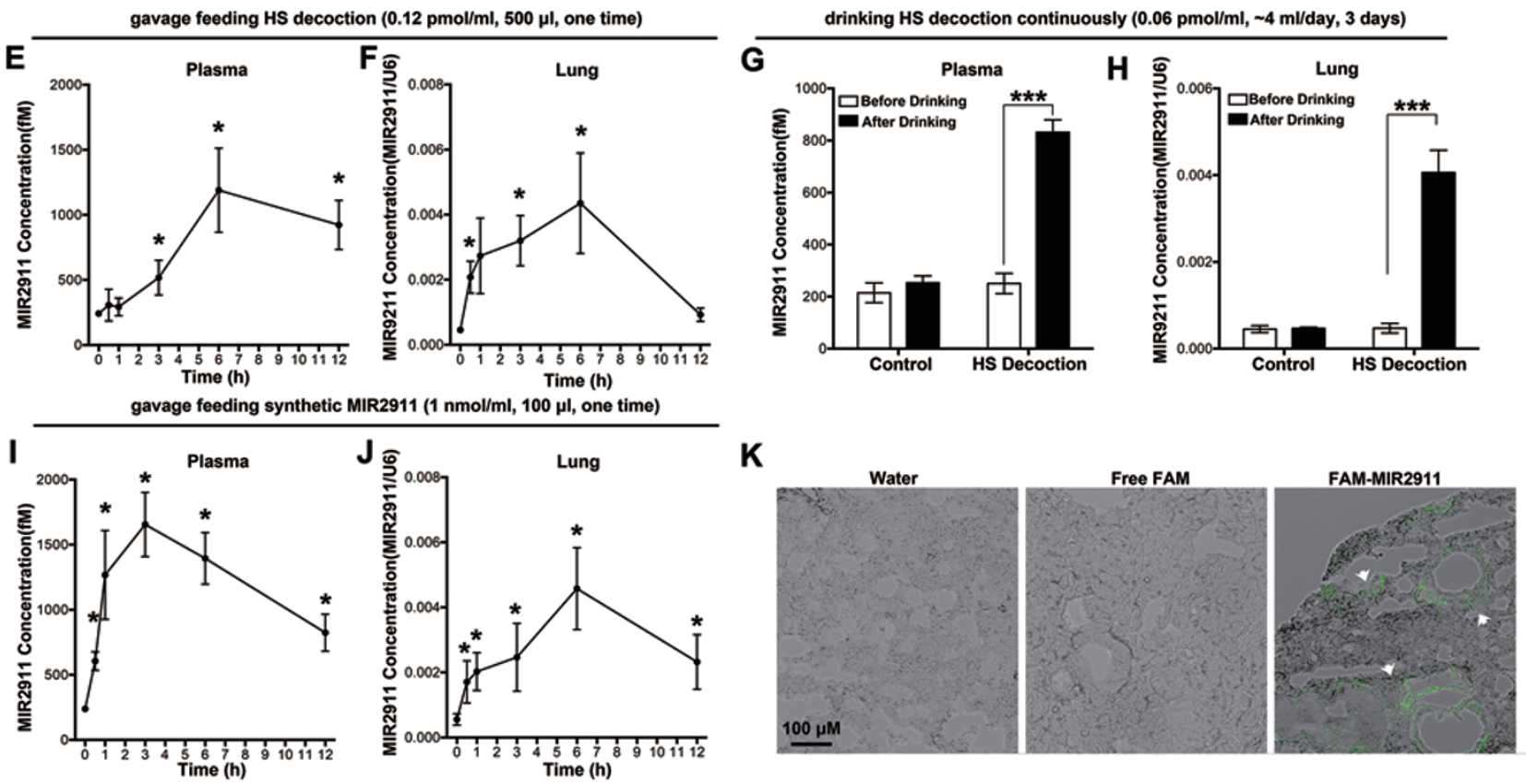

Figure 1 MIR2911 is highly enriched in HS decoction and is delivered into mouse lungs. (A, B) The levels (sequencing reads) of plant miRNAs in HS (A) and HS decoction (B) detected by Illumina sequencing. (C) The concentrations of MIR2911 in HS and HS decoction determined by RT-qPCR. (D) Northern blotting analysis showing MIR2911 expression in $10 \mathrm{~g}$ of HS or 10 $\mathrm{ml}$ of HS decoction (final concentration: $\sim 0.2 \mathrm{~g} \mathrm{HS} / \mathrm{ml}$ ). (E) MIR2911 kinetics in mouse plasma after administration of $500 \mu \mathrm{l}$ of HS decoction (MIR2911 concentration: $0.12 \mathrm{nM}$ ) by gavage $(0 \mathrm{~h}, n=30$; other time point, $n=6)$. (F) MIR2911 kinetics in mouse lung tissue after administration of $500 \mu \mathrm{l}$ of HS decoction (MIR2911 concentration: $0.12 \mathrm{nM})$ by gavage $(n=5)$. (G, H) The MIR2911 level in mouse plasma $(\mathbf{G})$ and lung tissue $(\mathbf{H}) 3$ days after drinking HS decoction (MIR2911 concentration: 0.06 $\mathrm{nM})(n=5)$. (I, J) The MIR2911 kinetics in mouse plasma (I) and lung tissue (J) after administration of 100 pmol synthetic MIR2911 in $100 \mu$ I PBS by gavage $(n=5)$. (K) Fluorescently labeled MIR2911 in mouse lung tissue $3 \mathrm{~h}$ after administration of $2 \mathrm{nmol}$ synthetic, fluorescently labeled MIR2911 by gavage (representative images are shown). Note that labeled MIR2911 (green dots) rapidly accumulated in mouse lungs. ${ }^{*} P<0.05$; ${ }^{* *} P<0.001$. All values are the mean \pm SEM. $P$ values were determined using Student's $t$-test.

$\pm 26.3 \mathrm{fM}$ ) (Figure 1G). The level of MIR2911 in mouse lungs after drinking HS decoction increased by $>8$-fold, whereas no change in the MIR2911 level was observed in control mouse lungs (Figure 1H). As shown in Supplementary information, Figure S4, an $\sim 10$-fold increase in MIR2911 level was also detected in the liver, while the levels of MIR2911 in the mouse intestines and kidneys remained unchanged. The concentration of MIR2911 in mouse lungs was nearly equal to that of endogenous miR-25 (Supplementary information, Figure S5). The uptake of MIR2911 through the mouse GI tract was further tested by administering synthetic MIR2911 to mice ( $1 \mathrm{nmol} / \mathrm{ml}, 100 \mu \mathrm{l}$ given once per mouse). Mice that were gavage-fed with sterile water served as controls. As shown in Figure 1I, the plasma levels of MIR2911 continually increased from $239.3 \pm 19.2 \mathrm{fM}$ to 1655.7 
$\pm 246.8 \mathrm{fM}$ within $3 \mathrm{~h}$ and then gradually decreased. At $12 \mathrm{~h}$, the plasma levels of MIR2911 decreased to 824.2 $\pm 141.7 \mathrm{fM}$. The level of MIR2911 in mouse lungs also continually increased, reached a peak level at $6 \mathrm{~h}$ and then gradually decreased (Figure 1J). In addition, the delivery of MIR2911 into mouse lungs was illustrated by the administration of a fluorescently labeled, synthetic MIR2911 by gavage. Bright fluorescent dots were observed in lung sections from mice treated with fluorescent MIR2911 (Figure 1K).

Plant MIR2911 directly binds to some types of IAVs and inhibits H1N1 virus-encoded PB2 and NS1 protein expression as well as H1N1 viral replication in vitro

To determine whether MIR2911 is an active anti-viral component in HS decoction, bioinformatics analysis using RNAhybrid [10] was performed to identify the sequences in influenza virus mRNA that bound to MIR2911. As shown in Figure 2A, MIR2911 has potential target genes in various IAVs. To verify whether MIR2911 can bind to the predicted target genes in H1N1, H3N2, H5N1 and H7N9 viruses, 80 bp nucleic acid fragments covering the predicted MIR2911-binding sites on various viral genes were inserted into a plasmid containing a luciferase reporter. Compared to ncRNA, MIR2911 reduced luciferase activity by $57.4 \% \pm 1.4 \%$, $53.1 \% \pm 4.3 \%, 52.1 \% \pm 2.5 \%, 66.4 \% \pm 2.4 \%$ and $71.2 \%$ $\pm 3.2 \%$ through binding to target sequences in H1-PB2, H1-NS1, H3-HA, H5-NP and H7-HA, respectively (Figure 2B). In contrast, the MIR2911-dependent inhibition of the luciferase activity of all luciferase reporters was abolished after mutation of the MIR2911-binding sites on the individual viral genes. Next, the direct targeting of the PB2 and NS1 genes of A/Sichuan/1/2009 (H1N1) [11] by MIR2911 was selectively tested. Two plasmids that expressed the $P B 2$ or $N S 1$ gene were constructed. The MIR2911-binding sequence in the $P B 2$ or NS1 gene was mutated without altering the amino acid sequence. MIR2911 strongly downregulated the protein levels of the wild-type $P B 2$ and NS1, while the expression levels of the $P B 2$ and NS1 mutants remained unchanged (Figure $2 \mathrm{C}$ and $2 \mathrm{D})$.

Given that $P B 2$ and NS1 genes are essential for influenza viral replication $[12,13]$, the inhibitory effects of MIR2911 on the replication of an H1N1 virus in Madin-Darby Canine Kidney (MDCK) cells were examined. A/Sichuan/1/2009 (H1N1), previously isolated from a patient in China [11], was used in the following experiments. As shown in Figure 2E, the viral titer of H1N1 was significantly decreased when virus-infected MDCK cells were transfected with synthetic MIR2911. The log value of $\mathrm{TCID}_{50} / \mathrm{ml}$ was reduced from $3.14 \pm 0.10$ to 2.21 \pm 0.22 at $12 \mathrm{~h}$ and from $5.43 \pm 0.10$ to $3.80 \pm 0.25$ at 24 $\mathrm{h}$ after transfection. In contrast, the viral titer of H1N1 was not affected by transfection with ncRNA (Figure 2E). A similar inhibitory effect on $\mathrm{H} 1 \mathrm{~N} 1$ replication was achieved by transfecting total RNA extracted from HS decoction into virus-infected cells (Figure 2F). The log value of $\mathrm{TCID}_{50} / \mathrm{ml}$ was reduced from $3.08 \pm 0.14$ to 2.30 \pm 0.25 at $12 \mathrm{~h}$ and from $5.60 \pm 0.03$ to $4.27 \pm 0.19$ at 24 $\mathrm{h}$ after transfection. The inhibitory effect of the HS decoction total RNA was abolished by co-transfection with the anti-MIR2911 antagomir, suggesting that MIR2911 is the active anti-viral component in HS decoction (Figure 2F). To further test the specificity of MIR2911-mediated inhibition of viral replication, the effect of MIR2911 or HS decoction on H1N1 replication, after mutating the MIR2911-binding sites in the PB2 and NS1 genes without changing the amino acid sequences, was assessed (Supplementary information, Figure S6). Replication of the mutant H1N1 virus was not inhibited by synthetic MIR2911 or total RNA extracted from HS decoction (Figure $2 \mathrm{G}$ and $2 \mathrm{H}$ ).

Plant MIR2911 rescues virus inoculation-induced weight loss and inhibits viral replication in H1N1-inoculated mice

The anti-viral effect of HS decoction and MIR2911 was further tested in H1N1-infected mice. In this experiment, 6-week-old female BALB/c mice were administered synthetic MIR2911 (0.1 nmol per day) by gavage or allowed to drink HS decoction one day before being inoculated with $10^{6} \mathrm{EID}_{50} \mathrm{H} 1 \mathrm{~N} 1$ virus. Following the virus inoculation, the mice were continually treated with MIR2911 or HS decoction for 7 days. As shown in Figure $3 \mathrm{~A}$, the mice infected with $\mathrm{H} 1 \mathrm{~N} 1$ alone or co-treated with H1N1 and ncRNA rapidly lost $20 \%$ of their weight on day 7 . In contrast, both administration of synthetic MIR2911 and continuous drinking of HS decoction effectively prevented weight loss $(<10 \%)$. The protection of weight loss caused by drinking HS decoction was completely abolished by using the anti-MIR2911 antagomir (Figure 3B). As shown in Figure 3C, the viral titers in the lung tissues from H1N1-infected mice on days 3 and 5 post infection were high $\left(\log \mathrm{EID}_{50}\right.$ of $6.0 \pm 0.16$ on day 3 and $\log \mathrm{EID}_{50}$ of $5.25 \pm 0.25$ on day 5), while administration of MIR2911 or drinking of HS decoction significantly reduced the viral titers $\left(\log \mathrm{EID}_{50}\right.$ of $4.42 \pm$ 0.22 or $4.50 \pm 0.09$, respectively, on day 3 and $\log \mathrm{EID}_{50}$ of $4.25 \pm 0.14$ or $4.08 \pm 0.15$, respectively, on day 5 ). Administration of ncRNA did not affect the viral titer (Figure 3C). Moreover, the inhibitory effect of HS decoction on virus duplication was completely reversed by the anti-MIR2911 antagomir (Figure 3C). To further assess the 
A

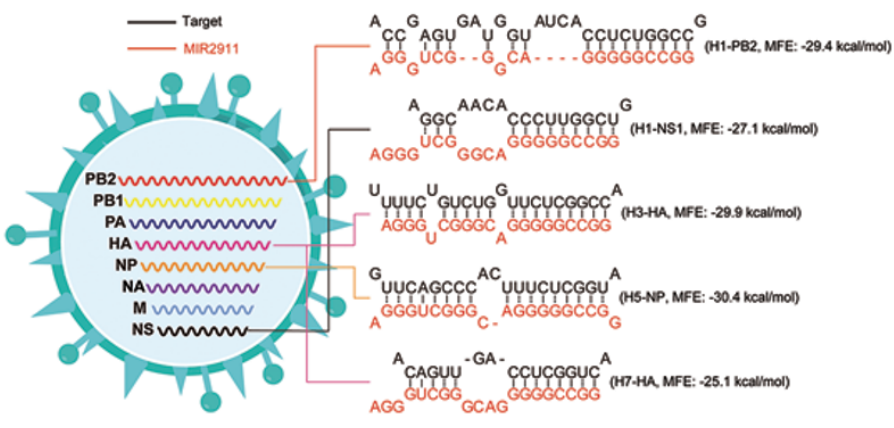

C

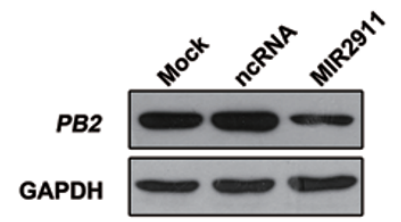

NS1

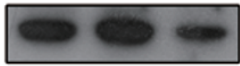

GAPDH

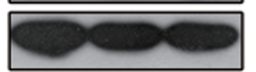

E

G
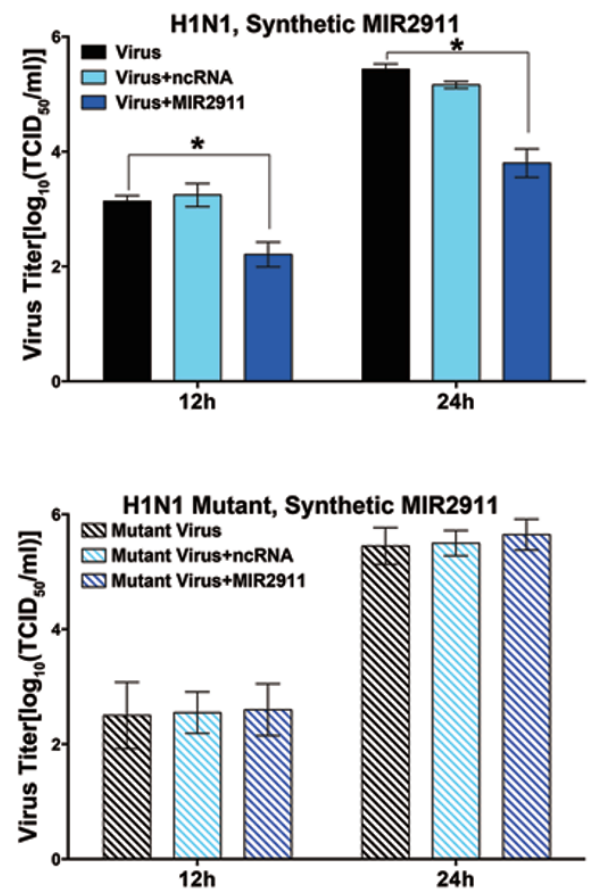

B

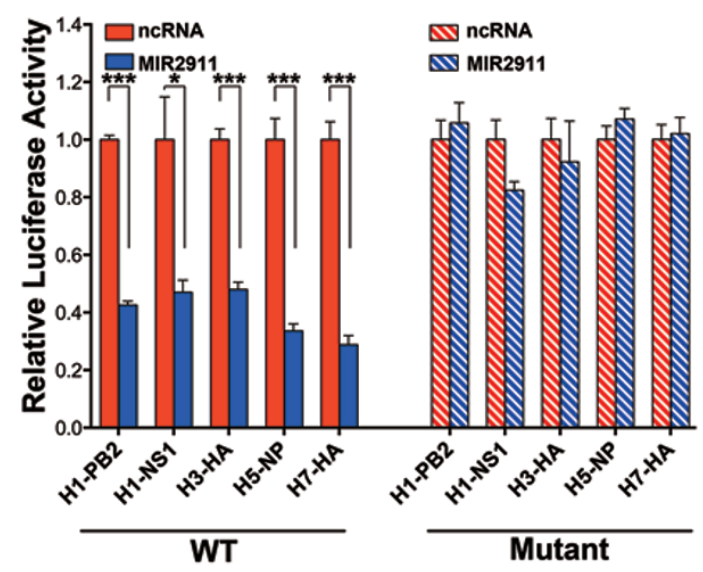

D

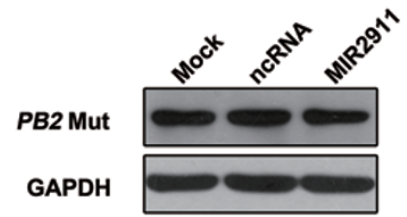

NS1 Mut

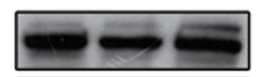

GAPDH

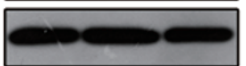

F

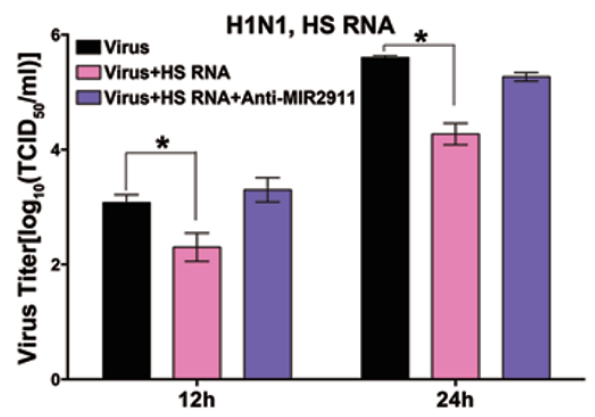

H

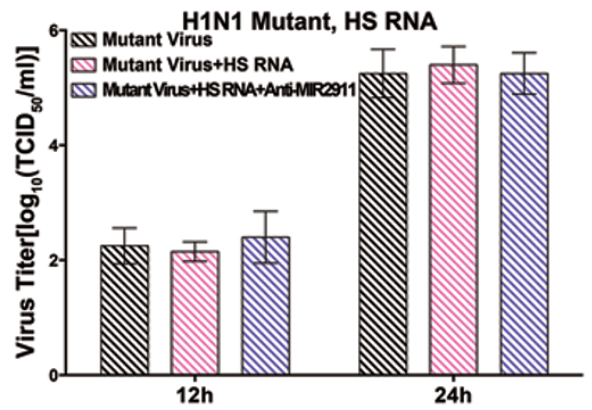

Figure 2 MIR2911 targets various influenza viruses. (A) Prediction of the viral genome sequence targeted by MIR2911. (B) Validation of the predicted MIR2911 target sequence using a luciferase reporter assay $(n=3)$. (C) Western blot analysis of the PB2 and NS1 levels after transfecting HEK293T cells with the plasmids expressing PB2 or NS1, MIR2911 or ncRNA. (D) Western blot analysis of the mutant PB2 and NS1 levels after transfecting HEK293T cells with the plasmids expressing mutant PB2 or NS1, MIR2911 or ncRNA. (E, F) Anti-H1N1 effects of synthetic MIR2911 (E) and HS total RNA (F) in MDCK cells $(\mathrm{MOI}=0.1, n=6) .(\mathbf{G}, \mathbf{H})$ Effects of synthetic MIR2911 $(\mathbf{G})$ and HS total RNA $(\mathbf{H})$ on mutant H1N1 virus in MDCK cells (MOI $=0.1, n=6) .{ }^{*} P<0.05 ;{ }^{* \star \star} P<0.001$. All values are the mean \pm SEM. $P$ values were determined using Student's $t$-test. 

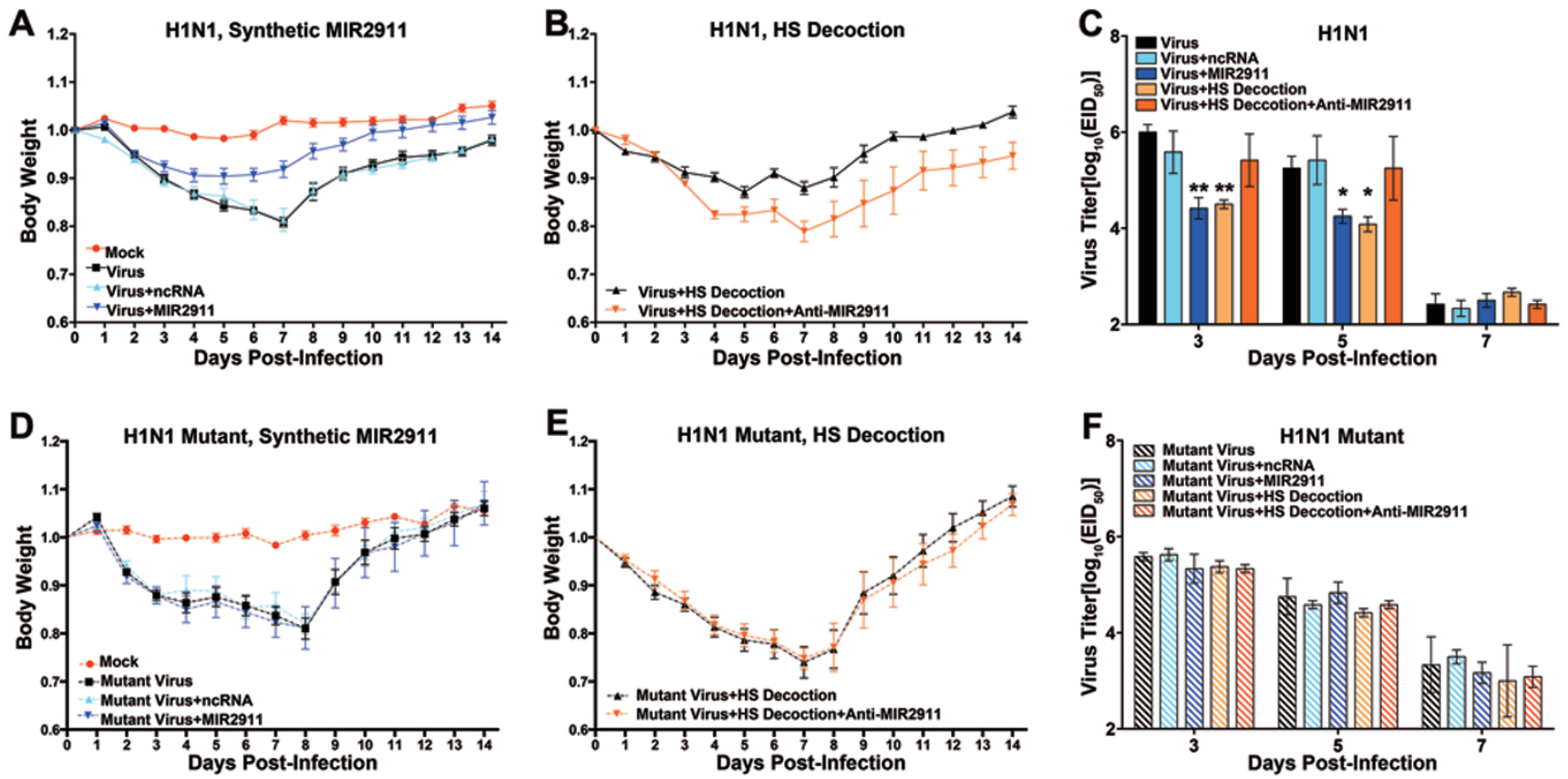

Figure 3 MIR2911 suppressed the replication of H1N1 influenza virus but not mutant virus in mice. The mice were treated with different reagents before being inoculated with H1N1 influenza viruses (A-C) or mutant H1N1 influenza virus (D-F). The body weight was recorded every day. On days 3, 5 and 7 post infection, the mice were sacrificed, and lung tissue was obtained to measure viral replication. (A, B) Body weight changes in mice subjected to synthetic MIR2911 (A) and HS decoction (B) treatment after inoculation with $10^{6} \mathrm{EID}_{50}$ of $\mathrm{H} 1 \mathrm{~N} 1$ influenza virus $(n=8)$. (C) Viral titers $\left(\mathrm{EID}_{50}\right)$ in the lungs of mice subjected to synthetic MIR2911 and HS decoction treatment at different times after inoculation with $10^{6}$ EID $_{50}$ of $\mathrm{H}_{1 \mathrm{~N} 1}$ influenza virus $(n=6)$. (D, E) Body weight changes in mice subjected to synthetic MIR2911 (D) and HS decoction (E) treatment after inoculation with $10^{6} \mathrm{EID}_{50}$ of mutant $\mathrm{H} 1 \mathrm{~N} 1$ influenza virus $(n=8)$. (F) Viral titers $\left(\mathrm{EID}_{50}\right)$ in the lungs of mice subjected to synthetic MIR2911 and HS decoction treatment at different times after inoculation with $10^{6} \mathrm{EID}_{50}$ of mutant H1N1 influenza virus $(n$ $=6)$. ${ }^{*} P<0.05 ;{ }^{* *} P<0.01$. All values are the mean \pm SEM. $P$ values were determined using Student's $t$-test by comparing to the virus alone group.

specificity of MIR2911 inhibition in vivo, mutant H1N1 virus $\left(10^{6} \mathrm{EID}_{50}\right)$, in which the MIR2911-binding sites in the PB2 and NS1 gene were mutated without changing the amino acid sequences (Supplementary information, Figure S6), was used to infect mice. As shown in Figure $3 \mathrm{D}$ and $3 \mathrm{E}$, mutant H1N1 also caused weight loss and had a high viral titer similar to that of wild-type H1N1. In contrast, neither synthetic MIR2911 nor HS decoction had effects on the weight loss and virus titer in mice infected with mutant H1N1 (Figure 3D-3F).

Plant MIR2911 inhibits H5N1 and H7N9 viral replication in vitro and in vivo and rescues H5N1 inoculation-induced mortality

The potential effect of MIR2911 on other influenza viruses was further tested. A/Anhui/2/05 (H5N1), a highly pathogenic virus isolated from human in China [14], was used as the viral model. As shown in Figure 4A and 4B, the H5N1 virus rapidly infected MDCK cells. However, when the virus was transfected with synthetic MIR2911
(Figure 4A) or total RNA extracted from HS decoction (Figure 4B), the replication of the virus in MDCK cells was significantly inhibited (the log value of $\mathrm{TCID}_{50} / \mathrm{ml}$ for synthetic MIR2911 was reduced from $4.11 \pm 0.20$ to $2.74 \pm 0.34$ at $12 \mathrm{~h}$ and from $7.41 \pm 0.08$ to $5.92 \pm 0.19$ at $24 \mathrm{~h}$ after transfection; the $\log$ value of $\mathrm{TCID}_{50} / \mathrm{ml}$ for total RNA extracted from HS decoction was reduced from $4.27 \pm 0.20$ to $2.58 \pm 0.24$ at $12 \mathrm{~h}$ and from $7.49 \pm$ 0.06 to $5.97 \pm 0.17$ at $24 \mathrm{~h}$ after transfection). In addition, the inhibitory effect of the HS decoction total RNA was abolished by co-transfection with the anti-MIR2911 antagomir (Figure 4B). The effect of plant MIR2911 on $\mathrm{H} 5 \mathrm{~N} 1$ inoculation-induced mortality was assessed. As shown in Figure 4C, 3 mice each were dead on days 9 and 10 post infection, and overall, only 2 mice $(2 / 8)$ survived out of a group of H5N1-infected mice. Control ncRNA had no effect on H5N1-induced mouse death $(2,3,1$ and 1 mice were dead on days $9,10,11$ and 12 , respectively, and no mice were alive at the end of the experiment) (Figure 4C). Surprisingly, administration of 

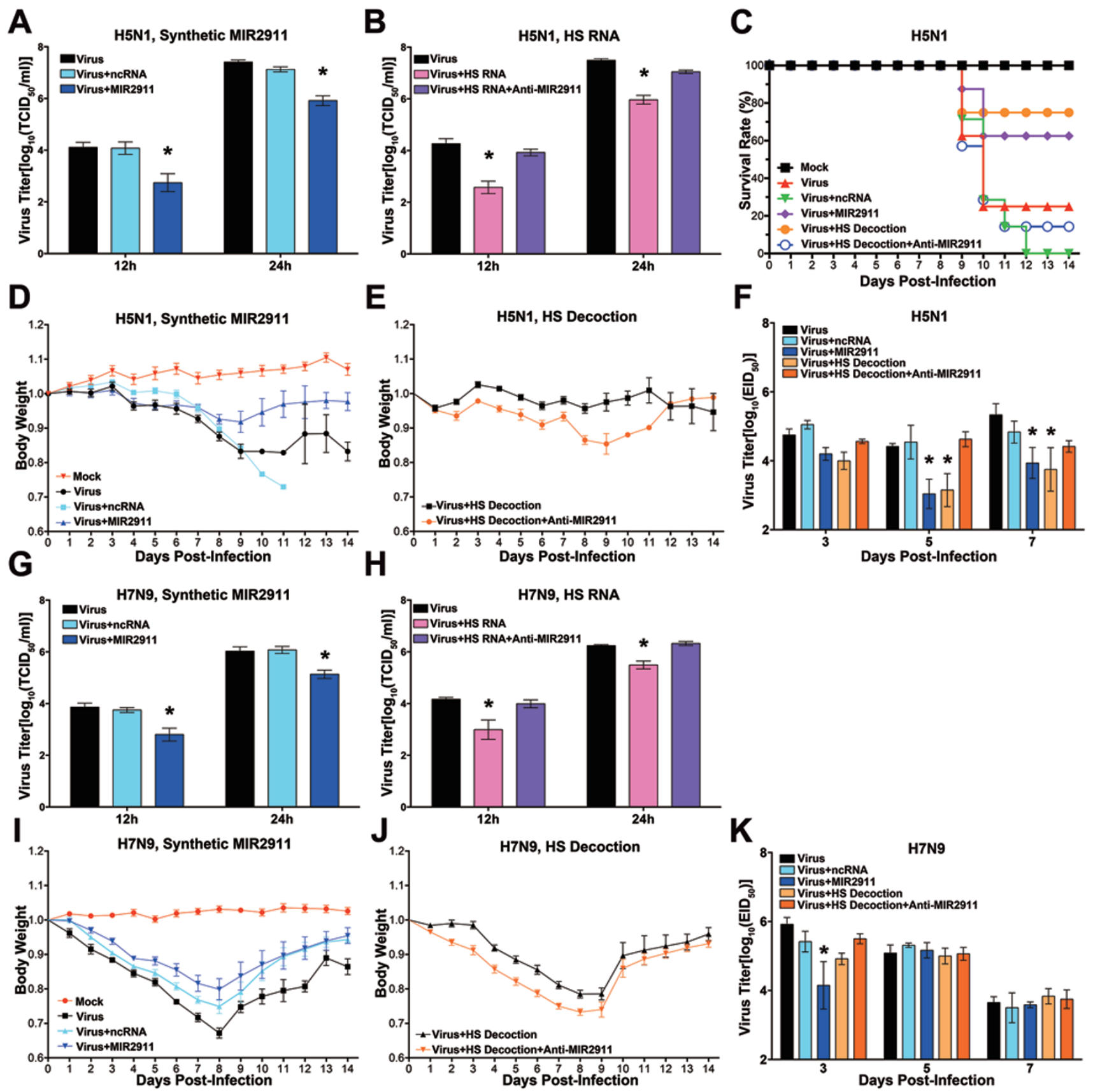

Figure 4 MIR2911 suppressed the replication of the H5N1 and H7N9 influenza viruses in mice. (A, B) The anti-H5N1 effects of synthetic MIR2911 (A) and HS total RNA (B) in MDCK cells (MOI =0.1, $n=6$ ). (C) Survival rates of mice subjected to synthetic MIR2911 and HS decoction treatment after intranasal inoculation with $10^{3}$ EID $_{50}$ of H5N1 influenza virus $(n=7-8)$. (D, E) Body weight changes in mice subjected to synthetic MIR2911 (D) and HS decoction (E) treatment after inoculation with $10^{3}$ EID $_{50}$ of H5N1 influenza virus $(n=7-8)$. $(\mathbf{F})$ Viral titers $\left(E_{50}\right)$ in the lungs of mice subjected to synthetic MIR2911 and HS decoction treatment at different times after inoculation with $10^{3} \mathrm{EID}_{50}$ of H5N1 influenza virus $(n=6)$. (G, H) The anti-H7N9 effects of synthetic MIR2911 (G) and HS total RNA (H) in MDCK cells (MOI = 0.1, $n=6)$. (I, J) Body weight changes in mice subjected to synthetic MIR2911 (I) and HS decoction (J) treatment after inoculation with $10^{6}$ EID $_{50}$ of H7N9 influenza virus $(n$ = 8). (K) Viral titers $\left(E I D_{50}\right)$ in the lungs of mice subjected to synthetic MIR2911 and HS decoction treatment at different times after inoculation with $10^{6} \mathrm{EID}_{50}$ of $\mathrm{H} 7 \mathrm{~N} 9$ influenza virus $(n=6) .{ }^{*} P<0.05$. All values are the mean \pm SEM. $P$ values were determined using Student's $t$-test by comparing to the virus alone group. 
synthetic MIR2911 and drinking of HS decoction significantly reduced $\mathrm{H} 5 \mathrm{~N} 1$-induced mortality (1 and 2 mice administered synthetic MIR2911 were dead on days 9 and 10 , respectively, and 5 mice $(5 / 8)$ survived to the end of the experiment; 2 mice that drank HS decoction were dead on day 9 post infection, and 6 mice (6/8) survived to the end of the experiment) (Figure 4C). Importantly, the anti-MIR2911 antagomir completely abolished the effect of HS decoction on mortality (3,2 and 1 mice were dead on days 9, 10 and 11, respectively, and only 1 mouse (1/7) survived to the end of the experiment) (Figure 4C). In addition, the body weight of mice infected with $\mathrm{H} 5 \mathrm{~N} 1$ alone or infected with $\mathrm{H} 5 \mathrm{~N} 1$ and treated with ncRNA was rapidly decreased, with a near $20 \%$ reduction on day 7 post infection (Figure 4D). Synthetic MIR2911 significantly rescued H5N1-induced weight loss $(<10 \%$ on day 9 post infection) (Figure 4D). Moreover, no significant weight loss was detected in mice that drank HS decoction (Figure 4E). Consistent with the results of the mortality analysis, the anti-MIR2911 antagomir completely abolished the effect of the HS decoction (Figure 4E). The viral titer of H5N1 in the lung tissues of H5N1-infected mice on days 5 and 7 post infection was significantly reduced by the administration of synthetic MIR2911 compared with similar administration of ncRNA $\left(\log\right.$ EID $_{50}$ reduced from $4.42 \pm 0.08$ to $3.04 \pm$ 0.49 on day 5 and from $5.34 \pm 0.32$ to $3.94 \pm 0.45$ on day 7) (Figure 4F). Drinking HS decoction also efficiently suppressed replication of the H5N1 virus in mouse lung tissues on days 5 and 7 post infection $\left(\log\right.$ EID $_{50}$ reduced from $4.42 \pm 0.08$ to $3.15 \pm 0.48$ on day 5 and from 5.34 \pm 0.32 to $3.75 \pm 0.63$ on day 7 ), whereas the inhibitory effect of HS decoction on H5N1 virus replication was completely abolished by depleting MIR2911 with anti-MIR2911 antagomir (Figure 4F).

The inhibitory effects of synthetic MIR2911 and HS decoction on the recently reported H7N9 influenza virus were also tested. A/Anhui/1/2013 (H7N9), previously isolated from a patient in China [5], was used in the following experiments. As shown in Figure $4 \mathrm{G}$ and $4 \mathrm{H}$, replication of the H7N9 virus in infected MDCK cells was strongly suppressed by synthetic MIR2911 or total RNA extracted from HS decoction (the log value of TCID $50 / \mathrm{ml}$ for synthetic MIR2911 was reduced from $3.86 \pm 0.16$ to $2.80 \pm 0.25$ at $12 \mathrm{~h}$ and from 6.02 \pm 0.17 to $5.13 \pm 0.16$ at $24 \mathrm{~h}$ after transfection; the $\log$ value of $\mathrm{TCID}_{50} / \mathrm{ml}$ for total RNA extracted from HS decoction was reduced from $4.17 \pm 0.08$ to $2.99 \pm 0.38$ at $12 \mathrm{~h}$ and from $6.24 \pm 0.04$ to $5.49 \pm 0.15$ at $24 \mathrm{~h}$ after transfection). The inhibitory effect of the HS decoction total RNA was again abolished by co-transfection with the anti-MIR2911 antagomir (Figure 4H). The effects of synthetic MIR2911 or HS decoction on weight loss in the H7N9-infected mice were also assessed. As shown in Figure 4I, mice treated with virus alone or H7N9 plus ncRNA showed nearly $30 \%$ or $20 \%$ weight loss, respectively, on day 8 post infection. Synthetic MIR2911 partially prevented H7N9-induced weight loss $(<20 \%$, Figure 4I). Similar to administration of synthetic MIR2911, drinking of HS decoction partially prevented H7N9 infection-induced weight loss (compared to Figure 4I, mice treated with virus alone or H7N9 plus ncRNA), while antagomir significantly blocked the effect of HS decoction on body weight (Figure 4J). The replication of the H7N9 virus in mouse lungs was also suppressed by synthetic MIR2911 or HS decoction on day 3 after H7N9 infection (Figure 4K).

\section{Discussion}

In Traditional Chinese Medicine, herbs are generally boiled for several hours to prepare the decoction. It is commonly believed that RNA will be destroyed during this process. Indeed, our data showed that most miRNAs (e.g., MIR166g and MIR2914) enriched in HS were degraded during the boiling process. However, a special miRNA, MIR2911, was found to be largely intact in the final HS decoction. MIR2911, previously reported as a plant miRNA in Populus euphratica, Nicotiana tabacum and Helianthus annuus [15-17], is an atypical miRNA because it is derived from ribosomal RNA (rRNA) and does not follow classical miRNA biogenesis [18-20]. Although the mechanism underlying the high stability of MIR2911 during the boiling process remains unknown, our data indicate that a unique sequence and high GC content may contribute to its stability. The resistance of MIR2911 to boiling process or even RNase treatment was abolished after the sequence was changed and GC content was decreased. The results reveal that miRNA may be an important, potentially effective but previously unrecognized component in Chinese herbs.

To exert an anti-viral effect against influenza in human body, MIR2911 in HS decoction must enter the circulation and accumulate in various organs particularly human lungs. Employing the mouse model, we demonstrated the delivery and accumulation of MIR2911 in various mouse organs: (1) Gavage feeding of HS decoction or synthetic MIR2911 led to a significant elevation of the MIR2911 level in mouse peripheral blood and lung tissues; (2) Continuous drinking of HS decoction led to a significant elevation of the MIR2911 level in mouse peripheral blood and lung; and (3) Gavage feeding of a fluorescently labeled, synthetic MIR2911 resulted in the detection of bright fluorescent dots in mouse lung sections. These 
results suggest that MIR2911 can pass through mouse GI tract and be transferred to the bloodstream and lungs, although the underneath mechanism remains unknown. Our previous study has suggested that intestinal epithelial cells can take up plant miRNAs in food, pack them into MVs in a form in association with AGO2 complex and then release them into the circulation; the AGO2-associated plant miRNAs encapsulated in MVs are then delivered into different tissues/cells in which the miRNAs modulate the expression of their target genes [9]. The majority of MIR2911 in mouse peripheral blood was detected in MV fraction, and MIR2911 in the MVs was largely associated with the AGO2 complex, supporting that MIR2911 executes its function through the same MV-mediated pathway. Our results indicate that the MIR2911 in HS decoction might be initially taken up through the GI tract, then repackaged into MVs by intestinal epithelial cells and finally delivered to lung through the circulatory system.

One of the question marks about the potential biological function of a plant miRNA in mammalian cells is the level of that exogenous miRNA. Because target encounter occurs by mass action and lowly expressed miRNAs have a less probability to meet target-containing transcripts [21], an miRNA expressed under a threshold concentration $(<100$ copies per cell $)$ is generally considered as noneffective. To ensure that the level of MIR2911 in mouse lungs is high enough to play a biological role, we calculated the amount of MIR2911 in mouse lung after gavage feeding with HS decoction. Given that each mouse lung contains 110-120 million cells [22], the average copy number of MIR2911 in each mouse lung cell reaches $300-400$, which is well above the minimal level required for miRNAs to execute their function. Consistent with this, we showed that the concentration of MIR2911 in mouse lungs was nearly equal to that of endogenous miR-25. Thus, MIR2911 fulfills the requirement for a threshold miRNA concentration. In support of this, in vivo study showed that MIR2911 could inhibit $\mathrm{H} 1 \mathrm{~N} 1, \mathrm{H} 5 \mathrm{~N} 1$ and $\mathrm{H} 7 \mathrm{~N} 9$ viral replication in mouse model, prevent viral infection-induced weight loss and even reduce mortality caused by infection.

In conclusion, the present study provides the first evidence that the highly stable plant MIR2911 can directly target multiple viral genes of various IAVs and thus suppress viral infections. With its broad-spectrum, anti-viral activity against IAVs, MIR2911 and MIR2911-containing HS decoction may represent a new effective therapeutic strategy that can be used to subdue deadly IAV infections. It is important to note that since Fleming discovered penicillin nearly a century ago, antibiotics have been developed to target various bacterial infections and have saved the lives of millions of people. Unfortunately, no natural product that is effective against viral infection has been identified thus far. We suggest that as the first natural product to directly target IAVs, MIR2911 is the "virological penicillin" that serves as a novel therapeutic and preventive agent against not only influenza A but potentially also other types of viruses.

\section{Material and Methods}

\section{Biosafety statement}

All experiments with live H5N1 and H7N9 viruses were conducted within the enhanced animal biosafety level 3 (ABSL3+) facility in the HVRI of the CAAS that was approved for such use by the Ministry of Agriculture of China and China National Accreditation Service for Conformity Assessment. All animal studies were approved by the Review Board of the HVRI, CAAS.

\section{Viruses}

The H1N1 virus A/Sichuan/1/2009 (SC/09) was isolated from the first human case of the 2009 influenza pandemic in China [11]. The H5N1 virus $\mathrm{A} / \mathrm{Anhui} / 2 / 2005(\mathrm{AH} / 05)$ was isolated from the respiratory specimens of a patient with lethal outcome from Anhui province in China in 2005 [14]. The H7N9 virus A/Anhui/1/2013 $(\mathrm{AH} / 13)$ was isolated from the respiratory specimens of a patient with lethal outcome from Anhui province in China in 2013 [5]. The SC/09 mutant was generated by reverse genetics. All rescued viruses were sequenced to exclude any unwanted mutations. Virus stock was propagated in specific pathogen-free chicken eggs.

\section{Honeysuckle}

HS was bought from a Chinese herbal medicine shop. HS decoction was prepared by boiling $10 \mathrm{~g}$ of HS in $100 \mathrm{ml}$ water for 30 min, resulting in $\sim 50 \mathrm{ml}$ decoction.

\section{Illumina deep sequencing}

Small RNAs were isolated from $1 \mathrm{~g}$ of HS or $5 \mathrm{ml}$ of HS decoction using the Universal Plant MicroRNA Kit (Bioteke, Beijing, China) according to the manufacturer's instructions. Illumina sequencing of RNA samples was performed by BGI (Shenzhen, China). After removing the adaptor sequences from the raw data, the clean reads were compared to known miRNA precursors and mature miRNAs from the miRBase database 14.0 to identify conserved plant miRNAs based on the Smith-Waterman algorithm. All data have been uploaded to the GEO database (GEO accession number: GSE55268).

\section{RNA isolation and RT-qPCR of mature miRNAs}

Small RNAs $(<100 \mathrm{nt})$ from HS and HS decoction were extracted using the Universal Plant MicroRNA Kit (Bioteke). Total RNA from plasma was extracted using the miRNeasy mini kit (Qiagen, Valencia, CA, USA), and total RNA from cells and tissues was isolated using TRIzol Reagent (Invitrogen, Carlsbad, CA, USA). Quantitative RT-PCR was performed using TaqMan miRNA probes (Applied Biosystems, Froster City, CA, USA) according to the manufacturer's instructions. Expression level of miRNAs in tissues was determined after normalization to U6 snRNA levels using the $2^{-\Delta \Delta C t}$ method. To calculate the absolute ex- 
pression levels of the target miRNAs, a series of synthetic miRNA oligonucleotides at known concentrations were reverse transcribed and amplified. The absolute amount of each miRNA was then calculated in reference to the standard curve. Quantitative PCR was performed using an ABI-7900 PCR machine.

\section{Northern blotting analysis}

Oligonucleotide probes complementary to mature miRNAs were end-labeled with $\gamma-{ }^{32} \mathrm{P}$-ATP using T4 Polynucleotide Kinase (Takara, Dalian, China), and labeled probes were purified using a Sephadex G25 spin column (Roche, Indianapolis, IN, USA). Small RNA was extracted from $10 \mathrm{~g}$ of HS or $10 \mathrm{ml}$ of HS decoction. RNA samples were fractionated by PAGE using a $15 \%$ denaturing polyacrylamide gel. The RNA was then transferred onto a nylon membrane (Hybond $\mathrm{N}^{+}$, Amersham Biosciences, Piscataway, NJ, USA) by electroblotting at $400 \mathrm{~mA}$ in $0.5 \times \mathrm{TBE}$ buffer for $1.5 \mathrm{~h}$. The membrane was cross-linked and dried. A prehybridization step was performed by incubating the membrane with $10 \mathrm{ml}$ of ULTRAhyb-Oligo solution (Ambion, Austin, TX, USA) at $37^{\circ} \mathrm{C}$ for $1 \mathrm{~h}$. The radiolabeled probe was added directly to the ULTRAhyb-Oligo solution, and the membrane was incubated overnight at $37{ }^{\circ} \mathrm{C}$ with rotation in a hybridization oven. After hybridization, the membrane was washed twice at low stringency in $2 \times \mathrm{SSC}, 0.1 \%$ SDS at $42{ }^{\circ} \mathrm{C}$ for $10 \mathrm{~min}$. The membrane was wrapped in a plastic wrap and exposed to an X-ray film at $-80^{\circ} \mathrm{C}$.

\section{MV isolation}

MVs were isolated from plasma by differential centrifugation according to previous publications [23]. Briefly, after removing cells and other debris by centrifugation at $300 \times g, 1200 \times g$ and 10 $000 \times g$, the supernatant was centrifuged at $110000 \times g$ for $2 \mathrm{~h}$ (all steps were performed at $4{ }^{\circ} \mathrm{C}$ ). MVs were collected from the pellet and resuspended in FBS-free medium.

\section{Immunoprecipitation}

MVs were resuspended in an appropriate volume of complete immunoprecipitation lysis buffer $(20 \mathrm{mM}$ Tris- $\mathrm{HCl}, \mathrm{pH} 7.5,150$ $\mathrm{mM} \mathrm{NaCl}, 0.5 \% \mathrm{NP}-40,2 \mathrm{mM}$ EDTA, $0.5 \mathrm{mM}$ dithiothreitol (DTT), $1 \mathrm{mM} \mathrm{NaF}, 1 \times$ protease inhibitor and $1 \times \mathrm{PMSF}$ ) for $30 \mathrm{~min}$ on ice. The lysates were immunoprecipitated with mouse monoclonal anti-AGO2 antibody or mouse normal IgG followed by protein G-Agarose beads. After purification, the immunoprecipitated RNA was extracted with miRNeasy Mini Kit (Qiagen) and analyzed by RT-qPCR using TaqMan miRNA probes (Applied Biosystems).

\section{Bioinformatics analysis}

Influenza virus genomes were collected from the Influenza Virus Resources at NCBI [24]. RNAhybrid [10] was used to scan potential targets of MIR2911 in the viral sequence following two rules. First, the minimal folding energy was below $-20 \mathrm{kcal} / \mathrm{mol}$. Second, the hybrid region between the miRNA seed region and viral sequence did not contain mismatches. An optional rule for target prediction required conservation of the putative binding sites among the same influenza A subtypes.

\section{Plasmid conduction and luciferase assay}

The binding sequences of the MIR2911 targets and the mutants (Invitrogen) were synthetically placed into the $3^{\prime} \mathrm{UTR}$ region of the pMIR-report plasmid (Ambion), and efficient insertion was confirmed by sequencing. For the luciferase reporter assays, $0.2 \mu \mathrm{g}$ of firefly luciferase reporter plasmid, $0.2 \mathrm{~g}$ of $\beta$-galactosidase expression vector (Ambion) and equal amounts $(20 \mathrm{pmol})$ of mature MIR2911 or ncRNA were transfected into cells in 24-well plates. The $\beta$-galactosidase vector was used as a transfection control. At $24 \mathrm{~h}$ post transfection, the cells were analyzed using a luciferase assay kit (Promega, Madison, WI, USA).

\section{Western blot analysis}

The PB2 or NS1 plasmids were co-transfected with synthetic MIR2911 or ncRNA into HEK293T cells using Lipofectamine 2000 (Invitrogen) according to the manufacturer's instructions. Samples of cultured cells were lysed in RIPA buffer $(0.5 \%$ NP-40, $0.1 \%$ sodium deoxycholate, $150 \mathrm{mM} \mathrm{NaCl}, 50 \mathrm{mM}$ Tris- $\mathrm{HCl}, \mathrm{pH}$ $7.5)$; the lysates were resolved by $10 \%$ SDS-PAGE, transferred to a PVDF membrane (Millipore, Bedford, MA, USA) and probed with anti-PB2, anti-NS1 or anti-GAPDH antibody (Santa Cruz Biotechnology, Santa Cruze, CA, USA).

\section{In vitro study}

MIR2911 or ncRNA was transfected into MDCK cells using Lipofectamine 2000 (Invitrogen) according to the manufacturer's instructions. Then, treated MDCK cells were infected with a virus at a multiplicity of infection of 0.1 at $37{ }^{\circ} \mathrm{C}$. After $1 \mathrm{~h}$ of incubation, the cells were washed with warm PBS and incubated in DMEM medium supplemented with $100 \mathrm{U} / \mathrm{ml}$ penicillin, $100 \mu \mathrm{g} /$ $\mathrm{ml}$ streptomycin, $2 \mu \mathrm{g} / \mathrm{ml}$ TPCK-treated trypsin and $0.2 \%$ bovine serum albumin fraction V. Supernatants were collected after 12 or $24 \mathrm{~h}$ and stored at $-70{ }^{\circ} \mathrm{C}$ for the $\mathrm{TCID}_{50}$ assay. In a separate experiment, MDCK cells were transfected with total RNA extracted from HS or from HS plus anti-MIR2911 antagomir. The treatments were performed as described above.

\section{Mouse study}

To study the kinetics of MIR2911 in mice, the mice were gavage fed with HS decoction (MIR2911 concentration: $0.12 \mathrm{nM}$ ) or $0.1 \mathrm{nmol}$ synthetic MIR2911. For HS decoction, plasma samples of five groups of mice ( 6 mice per group) were obtained prior to treatment as controls. Mice were then gavage fed with HS decoction. At each time interval $(0.5,1,3,6$ or $12 \mathrm{~h})$, mice were sacrificed, plasma samples were collected and total RNA was extracted. To collect tissue samples, six groups of mice ( 5 mice per group) including control group were sacrificed at the fixed time interval. For synthetic MIR2911, five groups of mice (5 mice per group) were gavage fed with $100 \mathrm{pmol}$ synthetic MIR2911. After a fixed time interval $(0.5,1,3,6$ or $12 \mathrm{~h})$, mice were sacrificed, plasma samples and tissue samples were collected. Plasma samples and tissue samples were also collected from mice without treatments to serve as controls.

In a separate experiment, two groups of mice were fed with HS decoction (MIR2911 concentration: $0.06 \mathrm{nM}$ ) or sterile water for 3 days, followed by plasma and tissue collection. Total RNA was extracted from these samples. An RT-qPCR was performed to detect the MIR2911 level in these samples.

To study the anti-viral effect of MIR2911 in mice, 26 mice of each group were fed with the HS decoction or HS decoction plus anti-MIR2911 antagomir for $72 \mathrm{~h}$. These mice were then lightly anesthetized with $\mathrm{CO}_{2}$ and inoculated intranasally with a $50 \mu \mathrm{l}$ volume of $10^{6} \mathrm{EID}_{50}$ of SC/09 (H1N1), $10^{3} \mathrm{EID}_{50}$ of AH/05 (H5N1) 
or $10^{6} \mathrm{EID}_{50}$ of $\mathrm{AH} / 13$ (H7N9). Mice were continually treated with HS decoction or HS decoction plus anti-MIR2911 antagomir (concentration: $20 \mathrm{nM}$ ) after inoculation. Eighteen mice $(n=6$ in each time point) were euthanized on days 3,5 or 7 post inoculation, and lungs were collected and titrated for virus infectivity in eggs. The remaining mice $(n=8)$ were monitored for 14 days for weight loss and mortality. In a separate experiment, each group of 26 mice was gavage fed with a $200 \mu \mathrm{l}$ volume of PBS, synthetic MIR2911 $(0.1$ nmol) or ncRNA $(0.1 \mathrm{nmol})$ per day for 8 days. On day 2, these mice were lightly anesthetized with $\mathrm{CO}_{2}$ and inoculated intranasally with a $50 \mu$ volume of $10^{6} \mathrm{EID}_{50}$ of SC/09 (H1N1), $10^{3} \mathrm{EID}_{50}$ of $\mathrm{AH} / 05(\mathrm{H} 5 \mathrm{~N} 1)$ or $10^{6} \mathrm{EID}_{50}$ of $\mathrm{AH} / 13$ (H7N9). Eighteen mice $(n=$ 6 in each time point) were euthanized on days 3,5 or 7 post inoculation, and lungs were collected and titrated for virus infectivity in eggs. The remaining mice $(n=8)$ were monitored for 14 days for weight loss and mortality.

\section{Acknowledgments}

We thank Dr Zheng-Gang Liu (Cancer Research Institute, NIH) and Dr Dan Wu (Yale University) for their comments on the manuscript. This work was supported by the National Basic Research Program of China (973 Program; 2014CB542300), the National Natural Science Foundation of China (81101330, 31271378 and 81250044), the Natural Science Foundation of Jiangsu Province (BK2011013, BK2012014 and BK20130592) and the Research Special Fund for Public Welfare Industry of Health (201302018).

\section{References}

1 Frost WH. Statistics of influenza morbidity: With special reference to certain factors in case incidence and case fatality. Public Health Rep (1896-1970) 1920; 35:584-997.

2 Guan Y, Vijaykrishna D, Bahl J, Zhu H, Wang J, Smith GJ. The emergence of pandemic influenza viruses. Protein Cell 2010; 1:9-13.

3 Chen H, Deng G, Li Z, et al. The evolution of H5N1 influenza viruses in ducks in southern China. Proc Natl Acad Sci USA 2004; 101:10452-10457.

4 Gao R, Cao B, Hu Y, et al. Human infection with a novel avian-origin influenza A (H7N9) virus. N Engl J Med 2013; 368:1888-1897.

5 Zhang Q, Shi J, Deng G, et al. H7N9 influenza viruses are transmissible in ferrets by respiratory droplet. Science 2013; 341:410-414.

6 Layne SP, Monto AS, Taubenberger JK. Pandemic influenza: an inconvenient mutation. Science 2009; 323:1560-1561.

7 Ko HC, Wei BL, Chiou WF. The effect of medicinal plants used in Chinese folk medicine on RANTES secretion by virus-infected human epithelial cells. J Ethnopharmacol 2006; 107:205-210.

8 Yang MS, Law FC, Wong RN, Mak NK, Wei XY. Interaction between oseltamivir and herbal medicines used for treating avian influenza. Hong Kong Med J 2012; 18 Suppl 6:34-36.

9 Zhang L, Hou D, Chen X, et al. Exogenous plant MIR168a specifically targets mammalian LDLRAP1: evidence of cross-kingdom regulation by microRNA. Cell Res 2012; 22:107-126.

10 Kruger J, Rehmsmeier M. RNAhybrid: microRNA target prediction easy, fast and flexible. Nucleic Acids Res 2006; 34:W451-W454.

11 Zhang Y, Zhang Q, Gao Y, et al. Key molecular factors in hemagglutinin and PB2 contribute to efficient transmission of the 2009 H1N1 pandemic influenza virus. J Virol 2012; 86:9666-9674.

12 Ishihama A. A multi-functional enzyme with RNA polymerase and RNase activities: molecular anatomy of influenza virus RNA polymerase. Biochimie 1996; 78:1097-1102.

13 Hale BG, Randall RE, Ortin J, Jackson D. The multifunctional NS1 protein of influenza A viruses. J Gen Virol 2008; 89:2359-2376.

14 Li Y, Shi J, Zhong G, et al. Continued evolution of H5N1 influenza viruses in wild birds, domestic poultry, and humans in China from 2004 to 2009. J Virol 2010; 84:8389-8397.

15 Li B, Yin W, Xia X. Identification of microRNAs and their targets from Populus euphratica. Biochem Biophys Res Commun 2009; 388:272-277.

16 Tang S, Wang Y, Li Z, et al. Identification of wounding and topping responsive small RNAs in tobacco (Nicotiana tabacum). BMC Plant Biol 2012; 12:28.

17 Barozai MY, Baloch IA, Din M. Identification of MicroRNAs and their targets in Helianthus. Mol Biol Rep 2012; 39:25232532.

18 Gregory RI, Yan KP, Amuthan G, et al. The Microprocessor complex mediates the genesis of microRNAs. Nature 2004; 432:235-240.

19 Lee Y, Ahn C, Han J, et al. The nuclear RNase III Drosha initiates microRNA processing. Nature 2003; 425:415-419.

20 Denli AM, Tops BB, Plasterk RH, Ketting RF, Hannon GJ. Processing of primary microRNAs by the Microprocessor complex. Nature 2004; 432:231-235.

21 Brown BD, Venneri MA, Zingale A, Sergi Sergi L, Naldini L. Endogenous microRNA regulation suppresses transgene expression in hematopoietic lineages and enables stable gene transfer. Nat Med 2006; 12:585-591.

22 Stone KC, Mercer RR, Gehr P, Stockstill B, Crapo JD. Allometric relationships of cell numbers and size in the mammalian lung. Am J Respir Cell Mol Biol 1992; 6:235-243.

23 Zhang Y, Liu D, Chen X, et al. Secreted monocytic miR-150 enhances targeted endothelial cell migration. Mol Cell 2010; 39:133-144.

24 Bao Y, Bolotov P, Dernovoy D, et al. The influenza virus resource at the National Center for Biotechnology Information. J Virol 2008; 82:596-601.

(Supplementary information is linked to the online version of the paper on the Cell Research website.)

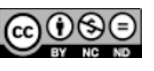
This work is licensed under the Creative Commons Attribution-NonCommercial-No Derivative Works 3.0 Unported License. To view a copy of this license, visit http:// creativecommons.org/licenses/by-nc-nd/3.0 